\title{
Utilização popular da Pterodon spp no tratamento de doenças reumáticas
}

\author{
The people use of Pterodon spp in treatment of rheumatic disease
}

\author{
Orestes Sérgio Amaral Bavaresco ${ }^{1}$, Ione Cristina de Paiva Pereira ${ }^{2}$, Cristiane Dominice \\ Melo $^{2}$, Felipe Lobato ${ }^{2}$, Angela Falcai ${ }^{2}$, Maria Rosa Quaresma Bomfim²
}

\begin{abstract}
Resumo: O presente estudo de revisão bibliográfica foi realizado entre dezembro de 2014 a maio de 2015, com abordagem descritiva e qualitativa dos dados, com a utilização de pesquisas em livros, revistas, jornais e artigos científicos na base de dados LILACS, Scielo e PubMed objetivou listar os nomes das plantas medicinais utilizadas no tratamento de doenças reumáticas. No Brasil, muitas pessoas sofrem de doenças reumáticas, as quais compreendem mais de 100 tipos diferentes de distúrbios, afetando, principalmente, as articulações, ossos, músculos esqueléticos, tendões e ligamentos. Os sintomas característicos são a inflamação das articulações, provocando, principalmente, dores lombares e nas pernas. Em geral, os alvos dessa patogenia são os idosos, devido a desgastes nas articulações; porém crianças, jovens e adultos também podem ser afetadas. $\mathrm{Na}$ flora medicinal, existem plantas usadas no tratamento do reumatismo, uma alternativa acessível e saudável para aqueles que acreditam na Fitoterapia. Os resultados evidenciaram a importância da utilização de partes da espécie vegetal Pterodon spp, para o tratamento de diferentes afecções reumáticas. As várias espécies de sucupira têm demonstrado importantes propriedades medicinais no tratamento de enfermidades inflamatórias (principalmente reumáticas), tendo sido comprovada a sua eficácia através de estudos químicos e farmacológicos. Os especialistas em produtos naturais recomendam a utilização moderada da sucupira indicando dosagens seguras para pacientes que fizerem uso de sua semente e óleo medicinal como faziam as pessoas antigamente.
\end{abstract}

Palavras-chave: Reumatismo. Fitoterapia. Plantas Medicinais. Pterodon

\begin{abstract}
This bibliographic review was conducted from December 2014 to May 2015, with descriptive and qualitative approach, with the use of research in books, magazines, newspapers and scientific articles in the LILACS database, Scielo and PubMed aimed list the names of medicinal plants used in the treatment of rheumatic diseases. In Brazil, many people suffer from rheumatic diseases, comprising more than 100 different types of disorders affecting primarily the joints, bones, skeletal muscles, tendons and ligaments. The characteristic symptoms are inflammation of the joints, resulting primarily in the legs and lower back pain. In general, the targets of this pathogenesis is the elderly, due to wear in the joints; But children, youth and adults can also be affected. In medicinal flora, plants are used in rheumatism treatment, affordable and healthy alternative for those who believe in herbal medicine. The results showed the importance of using parts of the plant species Pterodon spp, for the treatment of various rheumatic disorders. The various species sucupira have demonstrated significant medicinal properties in the treatment of inflammatory diseases (especially rheumatic), having been proven to be effective by chemical and pharmacological studies. Experts in herbal medicine recommend moderate use of sucupira indicating safe dosages for patients making use of their seed and medicinal oil, as did the people before.
\end{abstract}

Keywords: Rheumatism. Phytontherapy. Medicinal Plants. Pterodon

\footnotetext{
${ }^{1}$ Graduado do curso de Farmácia da Universidade Ceuma

${ }^{2}$ Docente da Universidade Ceuma
} 


\section{Introdução}

A utilização de plantas está inserida historicamente na vida do homem há muitos séculos ${ }^{1}$. Para expandir seu conhecimento a humanidade utilizou desde a préhistória sua intuição e analogia, fazendo assim, um caminho para descobrir a utilização de plantas para tratamentos específicos ${ }^{1}$. O homem ao observar os animais consumindo diferentes tipos de plantas foi aos poucos constatando os efeitos benéficos destas em seu organismo, e passou a ingeri-las também para se nutrir ${ }^{2}$. Paulatinamente foi descoberta a existência de muitas espécies que agiam de forma específica sobre as funções do corpo, ora ativando algumas, ora inibindo processos biológicos, com isso, o uso da flora medicinal expandiu-se e organizou-se em diversas sociedades ${ }^{2}$.

De acordo com a Organização Mundial da Saúde (OMS) as plantas medicinais são as principais fontes para se adquirir uma variedade de medicamentos, e em torno de $80 \%$ da população mundial utiliza a medicina tradicional para buscar alívio de alguma sintomatologia dolorosa ou desagradável ${ }^{3-6}$.

A seleção de plantas, a partir de suas informações da medicina tradicional ou popular, levou a descobertas promissoras da ação terapêutica para 0 ser humano ${ }^{7}$. Atualmente, várias plantas podem oferecer uma rica fonte de reserva para a descoberta de novos medicamentos contra inúmeras doenças, particularmente porque avanços nas técnicas de separação dos princípios ativos já estão disponíveis e têm conduzido a descoberta de uma variedade de plantas com potencial medicinal ${ }^{8,9}$.

$\mathrm{O}$ uso de plantas medicinais em pacientes portadores de doenças reumáticas tem despertado o interesse da comunidade científica ${ }^{10-14}$. Segundo Machado et al. $^{15}$ as doenças reumáticas são funções alteradas do sistema músculo esquelético de forma não traumática, tais como a artrite reumatoide, artrose, osteortrose e osteoartrite, sendo esta última a principal causadora de incapacidade física em adultos, em forma de dor e rigidez articular, dentre os fatores causadores das incapacidades, as doenças crônicas, em especial os reumatismos ou artropatias, merecem destaque, em virtude da sua alta prevalência na população idosa.Neste aspecto, por exemplo, algumas substâncias fitoterápicas já foram identificadas por apresentarem atividades contra artrite reumatóide (AR) moderada a grave, sendo à $A R$ uma doença autoimune inflamatória crônica e progressiva que afeta a qualidade de vida da maioria dos seus portadores, por acometer o tecido sinovial resultando em produção excessiva de líquido, destruição da cartilagem, erosão óssea adjacente e dano aos tendões e ligamentos 16,17 . Aproximadamente de 0,5 a $1 \%$ da população mundial é acometida por $\mathrm{AR}^{18}$, sendo mais comum em mulheres na proporção de $3: 1(\mathrm{M} / \mathrm{H})$. Sua prevalência aumenta com a idade, mas pode desenvolver-se também na infância ${ }^{19}$.

A busca por novos fármacos fitoterápicos tem estimulado a pesquisa clínica de várias espécies de plantas fortalecendo a confiabilidade na eficácia e segurança clínica da utilização de fitos para tratamento, alívio e cura de alguns tipos de doenças reumáticas, por esse modo, com a procura cada vez maior pelos produtos naturais, o Sistema Único de Saúde (SUS) oferece, desde 2010, medicamentos fitoterápicos na rede pública de saúde. Um deles, por exemplo, é à base da sucupira branca 
que é indicada para o tratamento de quadros reumáticos acompanhados de dor, como artrite reumatóide, fibromialgia, artrose e febre reumática ${ }^{20,21}$.

\section{Material e Método}

Trata-se de um estudo de revisão bibliográfica realizado entre dezembro de 2014 a maio de 2015, com abordagem descritiva, analítica e qualitativa dos dados, com a utilização de pesquisas em livros, revistas, jornais e artigos científicos na base de dados LILACS, Scielo e PubMed. Os seguintes descritores foram utilizados para a busca dos dados: Reumatismo, Fitoterapia e Plantas Medicinais.

De acordo com Lakatos ${ }^{22}$, a pesquisa descritiva visa conhecer as diversas situações e relações que ocorrem na vida social, política, econômica e demais comportamentos humanos individualmente, como de grupos e comunidades complexas.

\section{Revisão da literatura}

\section{Utilização de plantas para fins medicinais}

A preferência e utilização de produtos naturais, principalmente da flora, com fins de melhoria da saúde surgiram com a humanidade, indícios mostraram que 0 uso de plantas medicinais tóxicas foi encontrado em restos mortais em civilizações mais antigas, sendo considerada uma das práticas mais remotas utilizadas pelo homem para cura, prevenção e tratamento de doenças, servindo como importante fonte de compostos biologicamente ativos ${ }^{23}$. Nesse contexto, a natureza foi a primeira fonte de remédio e a primeira farmácia a que o homem recorreu ${ }^{2}$. O homem pré-histórico aprendeu através da observação a distinguir as plantas comestíveis e que trazia a cura, daquelas que podiam causar alguns efeitos indesejáveis ${ }^{24}$.

$\mathrm{Na}$ Idade Média o cultivo das ervas como fonte de alimentos, bebidas e remédios era a cargo dos monges, eles estudavam e decifravam as plantas que eram colhidas ao redor dos seus mosteiros e igrejas, fazendo assim, catálogos que serviriam para conhecimento posterior sobre a medicina alternativa ${ }^{25}$. A utilização de ervas de maneira empírica foi aos poucos sendo superada pelo uso da química experimental o que permitiu a síntese laboratorial de novas substâncias orgânicas. Este fato foi um importante passo para 0 surgimento da revolução industrial e tecnológica e a produção dos primeiros medicamentos sintéticos, uma vez que as doenças eram basicamente tratadas com plantas ou produtos derivados ${ }^{26,27}$.

Com o crescimento da indústria farmacêutica, o conhecimento e interesse sobre as plantas medicinais quase desapareceu, mas no fim do século XX aumentava cada vez mais a procura por plantas que podiam curar muitos males, pois havia ocorrido um abuso dos medicamentos sintéticos e à massificação das práticas médicas ${ }^{28}$.

Nas Américas os primeiros imigrantes trouxeram de seu país de origem mudas e sementes de suas ervas preferidas, que florescia junto com as ervas nativas ${ }^{29}$. Os primeiros registros sobre ervas medicinais na América foram feitas pelo médico mexicano Juan Badianus, em 1952, que retirou parte de seus conhecimentos da cultura indígena ${ }^{29}$. Historicamente, quando os portugueses chegaram ao Brasil eles encontraram índios que já faziam uso de ervas, tais como o urucum ou Bixa orellana que os nativos usavam para 
se proteger de picada de insetos, para tingir seus objetos e seus corpos em rituais $^{29}$.

Tratamento medicinal de afecções reumáticas

Vários estudos avaliando a atividade e a eficácia de algumas espécies de plantas medicinais no tratamento de enfermidades reumáticas têm sido conduzidos nos últimos anos. Contudo, é importante ressaltar que o reumatismo é um termo utilizado para mais de 200 tipos de doenças que atingem as articulações, as chamadas doenças reumáticas, entre as quais está à artrite reumatóide (AR) que é uma doença sistêmica inflamatória crônica caracterizada por dores articulares frequentes, com degradação das articulações atingidas e com deformidades irreversíveis ${ }^{14,20,30}$.

Por outro lado, a febre reumática, popularmente chamada de reumatismo no sangue, é uma patologia causada por bactérias do grupo dos Estreptococcos betahemolíticos que provoca inflamação nas articulações, afeta o sistema nervoso, destrói a cartilagem articular e óssea, afeta as válvulas cardíacas prejudicando o funcionamento do coração $^{30,31}$.

É importante ressaltar que o Brasil possui uma diversidade biológica bem vasta, sendo $10 \%$ dos 1,4 milhões de organismos vivos já descritos pela ciência são encontrados aqui, e mais de 55 mil espécies vegetais o que totaliza $22 \%$ da fauna em todo o planeta, logo, estudar as ervas brasileiras é estudar o país em sua dimensão continental, com toda a sua variabilidade do meio ambiente e do clima ${ }^{32}$.

Diversos tipos de plantas nativas do território brasileiro têm sido utilizadas no tratamento dos diferentes tipos de reumatismo, dentre estas têm se destacado o alecrim (Rosmarinus officinalis), cacto/mandacaru (Cereus jamacaru), chapéu de couro (Echinodorus macrophyllum), melão de São Caetano (Momordica charantia $L$ ), unha de gato (Ficus pumila) e a Sucupira (Pterodon spp.), esta última tem sido a mais utilizada. De acordo com Carvalho ${ }^{28}$ e Dutra ${ }^{33}$ o que pode ser utilizado para controlar artrite, artrose e reumatismo e que é natural pelo seu uso tradicional, para este tipo de controle é a semente de sucupira branca ou Pterodon emarginatus Vogel. Neste contexto, deu-se maior ênfase a três espécies do gênero Pterodon popularmente conhecida como sucupira, por ser a mais atuante em relação aos efeitos antiinflamatórios e de combate à dor no tratamento ou cura de alguns tipos de reumatismo.

\section{O gênero Pterodon}

O gênero Pterodon pertence à família Fabaceae, também chamada de Leguminosa que são Angiospermas de grande distribuição com aproximadamente $\quad 18.000$ espécies $^{34}$. Diferentes espécies têm utilização popular no tratamento de algumas enfermidades reumáticas, como por exemplo a Pterodon emarginatus Vogel. Esta espécie é popularmente conhecida como sucupira branca ou faveiro, árvore aromática nativa do cerrado brasileiro, que pode atingir de 5 a 15 metros de altura. Ela tem seu crescimento lento, mas é importante em reflorestamentos mistos em florestas para recuperar áreas degradadas, devido a sua tolerância para luz solar direta e de baixa fertilidade ${ }^{35}$. Exemplares Pterodon spp. são encontrados em áreas de transição se aproximando da região de floresta semi-decídua, mas 
podem também estar distribuídos por diferentes Estados brasileiros, tais como: Goiás, Minas Gerais e em São Paulo, onde encontra-se atualmente na lista de espécies ameaçadas ${ }^{33}$.

\section{Usos da sucupira na medicina popular}

A Farmacopéia Brasileira de 1929 refere o uso da casca de sucupira em forma de extrato fluido e tintura, citando a espécie como Bowdichia virgilioides Humboldt, Bonplant, Kunth ${ }^{34}$. A casca é retirada em grandes pedaços planos ou levemente curvos, de comprimento e largura variáveis entre $8-10 \mathrm{~mm}$ de espessura, a parte suberosa se desprende facilmente, expondo o parênquima cortical pardoavermelhado, sua face interna é amarelada com estrias longitudinais bem visíveis e seu sabor é amargo e adstringente ${ }^{33}$.

Estudos comprovaram que as sementes de sucupira da espécie Pterodon emarginatus possuem flavonoides, cumarinas, saponinas, triterpenos, esteróides e óleo essencial, em seu óleo é encontrado elementos como o beta-cariofileno, substância química com atividade antiinflamatória, antibiótica, antioxidante, anticarcinogênico e anestésico local, o que comprova as propriedades medicinais da Sucupira ${ }^{36}$.

O fruto da sucupira possui endocarpo, alado, rico em óleo, cuja fração volátil detém apreciáveis propriedades contra a penetração das cercarias $^{37,38}$. Na medicina, o óleo essencial de $P$. emarginatus, fortemente aromático, é usado no combate ao reumatismo e diabetes. Esse óleo amargo, quando misturado com água, é também empregado sob a forma de gargarejo, trazendo resultados positivos contra a inflamação da garganta em humanos ${ }^{36}$.
Com relação a indicação terapêutica, efeitos colaterais, contraindicações e riscos para o consumo de sucupira Carvalho ${ }^{28}$ descreveu que a sucupira funciona como depurador do sangue, excelente para articulações, combate úlceras, gastrites, ácido úrico, aftas, amidalite, artrite, artrose, asma, blenorragia, dermatoses, dor espasmódica, diabete, rouquidão, sífilis, hemorragias, vermes intestinais, inflamações no útero e ovário e também como anticancerígeno.

A sucupira é considerada uma planta muito segura para o consumo e não há estudos suficientes publicados correlacionados aos efeitos colaterais dessa espécie vegetal, também não foi encontrada nenhuma contraindicação quanto ao uso contínuo do extrato ou da tintura de sucupira ${ }^{28,39}$. No entanto, algumas pessoas recomendam que o chá de sucupira seja consumido por até quinze dias consecutivos, retomando seu uso após descansar por outros quinze dias, para que ocorra um equilíbrio no sistema endócrino ${ }^{39}$.

\section{Resultados e Discussão}

Após triagem de títulos e resumos de estudos identificados pela estratégia de busca com as palavraschave: Reumatismo, Fitoterapia e Plantas Medicinais, 101 publicações potencialmente relevantes foram selecionados e analisados mais detalhadamente suas informações, mas apenas 53 mostraram ser de grande relevância no assunto.

Estudos fotoquímicos em exemplares do gênero Pterodon spp. têm demonstrado a presença de alcalóides nas cascas das árvores ${ }^{40}$, isoflavona e alguns triterpenos ${ }^{41}$; diterpenos na madeira ${ }^{42,43}$ e isoflavona no óleo das sementes ${ }^{44}$. Apesar de existirem estudos sobre a atividade 
citotóxica de alguns diterpenos ${ }^{42}$ e sesquiterpenos $^{45}$ existem poucos estudos toxicológicos a respeito do óleo essencial, de Pterodon emarginatus Vogel ${ }^{26}$.

Pesquisas Químicas Biológicas e Agrícolas confirmaram as propriedades curativas de sucupira e a identificação de substâncias bioativas presentes nos extratos ${ }^{31}$. Para Foglio ${ }^{39}$ pessoas que fazem uso do chá de sucupira podem, ainda não terem tido nenhum problema de saúde, já que as substâncias usadas na bebida não são solúveis em água. Por outro lado, uma concentração muito pequena das propriedades da semente, podem não causar toxidade aguda, mas também nenhum benefício.
Alguns estudos têm mostrado a ação de diferentes partes da sucupira sobre 0 organismo com funções especificas, assim como suas doses diárias (Tabela1).

Silva et al. $^{47}$ demonstraram a atividade tanto anti-edematogênica tópica quanto aguda no extrato oleoso e em fração que continha esquiterpenos de $P$. pubescens. Enquanto Sabino et al. ${ }^{46}$ encontraram no extrato hidroalcoólico das sementes $P$. pubscens, atividade antiartrite. Em uma análise posterior sobre 0 estudo de Silva et al. ${ }^{48}$, Coelho et al. $^{50}$ revelaram que a sucupira havia efeitos anti-inflamatório e efeitos anti-nociceptivos no extrato etanóico da $P$. pubescens na forma de semente.

Tabela 1. Atividades biológicas in vivo do extrato da Pterodon spp.

Pterodon emarginatus ${ }^{1}$ / Pterodon polygalaeflorus ${ }^{2}$ / Pterodon pubescens $^{3}$

\begin{tabular}{|c|c|c|c|}
\hline Tratamento & Dose & Atividade farmacológica & Referência \\
\hline EHA & 5 à $50 \mathrm{mg} / \mathrm{kg}$ & $\begin{array}{c}\text { Antirreumática/antiartrítica } \\
\text { (3) }\end{array}$ & $\begin{array}{l}\text { Sabino et } \\
\text { al. } 46,47\end{array}$ \\
\hline $\mathrm{EH}$ & $500 \mathrm{mg} / \mathrm{kg}$ & $\begin{array}{c}\text { Anti-inflamatório } \\
(1,2)\end{array}$ & $\begin{array}{l}\text { Carvalho et } \\
\text { al. }^{49}\end{array}$ \\
\hline EO & $\begin{array}{c}40 \mu \mathrm{g} ; 200 \mu \mathrm{g} \\
\text { e } 200 \mathrm{mg} / \mathrm{kg}\end{array}$ & $\begin{array}{c}\text { Anti-inflamatório } \\
\text { (3) }\end{array}$ & Silva et al. ${ }^{48}$ \\
\hline OE & $100-1300 \mu \mathrm{g} / \mathrm{mL}$ & $\begin{array}{l}\text { Efeitos sobre contrações } \\
\qquad(1,2)\end{array}$ & $\begin{array}{l}\text { Evangelista et } \\
\text { al. }{ }^{53}\end{array}$ \\
\hline $\mathrm{EE}$ & $0.1 / 0.3 \mathrm{a} 1 \mathrm{~g} / \mathrm{kg}$ & $\begin{array}{l}\text { Anti-inflamatório/anti- } \\
\text { nociceptivo } \\
\text { (3) }\end{array}$ & Moraes $^{52}$ \\
\hline OE & $100-300 \mu \mathrm{g} / \mathrm{mL}$ & $\begin{array}{c}\text { Antiespasmódico } \\
\qquad(1,2,3)\end{array}$ & $\begin{array}{l}\text { Leonhardt et } \\
\text { al. }^{54}\end{array}$ \\
\hline
\end{tabular}


Na Tabela 1 é possível observar que dentre as apresentações dos extratos da Pterodon spp. a grande maioria mostrou ação direta sobre a dor e inflamação. O extrato alcoólicos dos frutos da Pterodon spp são utilizados na medicina popular como anti-inflamatórios, principalmente contra reumatismo, dores de garganta e analgesia em geral ${ }^{49}$. Os óleos dos frutos de pelo menos três espécies têm ação protetora contra o reumatismo, possuindo atividade antiinflamatória, fato este que motivou 0 início das pesquisas com estas espécies ${ }^{47,49-51}$.

\section{Conclusâo}

Os resultados do presente estudo mostraram que algumas espécies do gênero Pterodon, comumente conhecida como sucupira, tiveram a sua eficácia comprovada através de estudos químicos e farmacológicos para o tratamento de enfermidades inflamatórias (principalmente as reumáticas). Devido a isso, essa planta é tão comercializada nas feiras e mercados do país para tratamento de artrite, artrose, reumatismo, anti-inflamatório, cicatrizante e ação analgésica. A utilização de produtos naturais de origem vegetal, por ser de baixo custo, pode se tornar uma alternativa para maioria das pessoas, principalmente da camada mais carente da sociedade.

\section{Referências}

1. Di Stasi, I. C.; Hiruma-Lima, A. Plantas medicinais na Amazônia e na Mata Atlântica 2. ed. São Paulo: Editora UNESP, 1996.

2. Araújo, L. A medicina popular. 3.ed. Natal: Editora da UFRN, 2009.

3. Nascimento, G.G.F.; Locatelli, J.; Freitas, P.C.; Silva, G.L. Antibacterial activity of plant extracts and phytochemicals on antibiotic-resistant bacteria. Braz J Microbiol., v.31, p.247-256, 2000.

4. Caetano, N.;Saraiva, A.; Pereira, R.; Carvalho, D.; Pimentel, M. C. B.;Maia, M.B.S. Determinação de atividades antimicrobiana de extratos de plantas de uso popular como antiinflamatório.Rev.

Bras.

Farmacogn., v.12, p.132-135, 2002

5. Corrêa, M. F. P.; Melo, G. O.; Costa, S.S. Substâncias de origem vegetal potencialmente úteis na terapia da Asma. Rev. Bras. Farmacogn.,v.18, p.785797; 2008.

6. Sousa, F.C.F.; Melo, C.T.V.; Citó, M.C.O.; Félix, F.H.C.; Vasconcelos, S.M.M.; Fonteles, M.M.F.; Barbosa-Filho, J.M.; Viana, G.S.B. Plantas medicinais e seus constituintes bioativos: Uma revisão da bioatividade e potenciais benefícios nos distúrbios da ansiedade em modelos animais. Rev. Bras. Farmacogn., v. 18, p.642-654, 2008.

7. Agra M. F.;França P.F.;BarbosaFilho J. M. Synopsis of the plants known as medicinal and poisonous in Northeastof Brazil. 
Rev. Bras. Farmacogn., v.17, p.114-140; 2007.

8. Kitazato, K.; Wang, Y.; Kobayashi, N. Viral infectious disease and natural products with antiviral activity. Drug. Discov. Ther, v.1, n.1, p14-22. 2007.

9. Chattopadhyay, D.; Naik, T.N. Antivirals of ethnomedicinal origin: structure-activity relationship and scope. Mini Rev Med Chem, v.7, n.3, p.275301.2007.

10. Ernst, E. Complematary and alternative medicine in rheumatology. Bailliëreís Clin. Rheumatol.,v.14, n.4, p.731-49, 2000.

11. Aceves-Avila, F.J.; Medina, F.; Fraga, A. Herbal therapies in rheumatology: The persistence of ancient medical pratices. Clin Exp Rheumatol. v.19, p.177-183, 2001.

12. Jacobs, J.W.C.; Rasker, J.J.; Bijlsma, J.W.J. Alternative medicine in rheumatology: Threat or challenge? Clin. Exp. Rheumatol., v.19, p.117-119, 2001.

13. Soeken, K.L.; Miller, S. A.; Ernst, E. Herbal Medicines for the treatment of rheumatoid arthritis: a systematic review.Rheumatology.,v.42, p.652-659, 2003.

14.Setty, A.R.; Sigal, L. H. Herbal Medications Commonly Used in the Practive of Rheumatology: Mechanisms of Action, Efficacy, and Side Effects. Semin. Arthritis Rheum. v.34, n.6,p. 773-84, 2005.

15. Machado, G. P. D. M.; Barreto, S. M.; Passos V. M. D. A.; LimaCosta, M. F. F. Projeto Bambuí: prevalência de sintomas articulares crônicos em idosos. Rev Assoc. Med. Bras,v. 50, n. 4, p. 367-372, 2004.

16.Rosa, C.; Machado, C.A. Plantas medicinais utilizadas no tratamento de doenças reumáticas.Rev. Brasil. Farm. v. 88, p.26-32, 2007.

17. Mota, L.M.H.; Cruz, B.A.; Brenol, C. V.; Pereira, I. A.; RezendeFroza, L. S.; Bertolo, M. B.; Freitas, M. V.C.; Silva, N. A.; Louzada-Junior, P.; Giorgi, R.D. N.; Lima, R. A. C.; Kairalla, R. A.; Kawassaki, A. M.; Bernardo, W. M.; Pinheiro, G. R. C. Guidelines for the diagnosis of rheumatoid arthritis.Rev. Bras. Reumatol., v.53, n.2, p. 141-157, 2013.

18.Davies, A.; Cifaldi, M. A.; Segurado, O. G.; Weisman, M. $H$. Cost Effectiveness of Sequential Therapy with Tumor Necrosis Factor Antagonists in early Rheumatoid Arthritis. J. Rheumatol, v. 36 , n. 1, p. 16-24, 2009.

19.Bertolo, M. B. Artrite Reumatóide. Rev. Bras. Medicina, v. 65, n. 2, dez, 2008.

20. Brasil, Ministério da Saúde. Secretaria de Ciência, Tecnologia e Insumos Estratégicos. Departamento de Assistência Farmacêutica. A fitoterapia no SUS e o Programa de pesquisa de plantas medicinais da Central de Medicamentos. Brasília, 2006.

21. Boletim GPUIM - Grupo de Prevenção ao Uso Indevido de Medicamentos:Artrite

Reumatóide. Universidade Federal do Ceará, Fortaleza Ceará, v. 1, n. 01, 2011. 
22. Lakatos, E. M. Técnicas de pesquisa: planejamento e execução de pesquisas, amostragens e técnicas de pesquisa, elaboração, análise e interpretação de dados. 5. ed. São Paulo: Atlas, 2003.

23. Andrade, S.F.; Cardoso, L.G.; Bastos, J.K. Anti-inflammatory and antinociceptive activities of extract, fractions and populnoic acid from bark wood of Austroplenckia populnea. J. Ethnopharmacoly, v.109, n. 3, p. 464-471, 2007.

24. Franceschini-Filho, S. Plantas terapêuticas. São Paulo: Editora Organizações Andrei, 2004.

25. Gaspar, L. Plantas Medicinais.Pesquisa Escolar Online, Fundação Joaquim Nabuco, Recife. (2009) Disponível em: $<$ http://basilio.fundaj.gov.br/pesq uisaescolar/>. Acesso em: 15 de abr. 2015.

26. Da-Luz-Dias, F.; Takahashi. C. S.; Sakamoto-Hojo, E. T.; Vichinewski, W.; SARTI, S.J. Genotoxicity of the natural carcaricides "Sucupira" oil and era manthine in mammanlian cells in vitro and in vivo. Envornm. Molec Mutagen., v.26, p.338-344, 1995.

27.Dias, M. A.;Campos, A. H.; Cechinel, V. F.; Yunes, R. A.; Calixto, J. B. Analysis of the mechanisms underlying the contract response induced by hydroalcoholic extract of Pbyllantbus urinaria in the guinea-pig urinary in-vitro. J. Pharm. Pharmacol, v.47, n.10, p.846-51, 2002.

28. Carvalho, A.M.; Bonila, M.G.O.; Rieder, A.; Silva, P.L.; Costa,
V.A.E.; Amaral, A. C.; Araújo, G.G.; Ramo, P.R.; Ferreira, C.P.; Pereira, E.S. Plantas medicinais utilizadas no tratamento de doenças reumáticas na região Sudoeste de Mato Grosso, Brasil.(2004) (Univ. do Estado de Mato Grosso - UNEMAT, 2004.

29. Banóski. S. A. Ervas Medicinais. 2006. Disponível em: <http://www.atenas.edu.br/faculd ade/arquivos/NucleolniciacaoCie ncia/RevistaCientifica/REVISTA \%20CIENTIFICA\%202008/3\%20 ERVAS\%20MEDICINAIS\%20$\% 20$ Solange\%20Aparecida\%20 Banoski.pdf.> Acesso em 20 de fev.2015.

30. Brenol, C. V.; Monticielo, O. A.; Xavier, R. M.; Brenol, J. C. T. Artrite reumatóide e Aterosclerose. Rev. Assoc. Med. Bras., v.53, n.5, p. 465-70, 2007.

31. Netto, C.G. Pesquisas do CPQBA confirmam propriedades curativas da sucupira. Jornal da UNICAMP. Campinas 3 a 9 de novembro de 2008. Ano XXIII. n. 415.

32. Mittermeier, R. A.; Schwarz, M. \& Ayres, J. M.A. New species of marmoset, genus Callithrix $\mathrm{E}$ 1777 (Callitrichidae, Primates), from the Rio Maués region, state of Amazonas, Central Brasilian Amazonia. Goldiana Zool, v. 14, p. 1-17, 1992.

33. Dutra, R.C. Avaliação fitoquímica e farmacológica das sementes de Pterodon emarginatus. 2008. 244f.Dissertação (Mestrado do Curso de Pós-Graduação em Ciências Biológicas) UNIVERSIDADE FEDERAL DE JUIZ DE FORA. Juiz de Fora, 2008 
34. Souza, V.C.; Lorenzi, H. Botânica Sistemática - Guia ilustrado para identificação das famílias de angiospermas da flora brasileira. São Paulo: Instituto Plantarum de Estudos da Flora, 2005. ISBN9788586714214.

35. Hansen, D.; Haraguchi, M.; Alonso, A. Propriedades farmacêuticas de "sucupira" (Pterodon spp.). Braz. J. Pharm. Sci., v 46, n. 4 , dezembro de 2010.

36. Brandão, M. J. P.; LacaBuendia, J. F.; Macedo. Árvores nativas e exóticas do Estado de Minas Gerais. Informe Agropecuário, 23 (217): 264265, 2002.

37. Almeida, D. M. R. F.; RegitanoD'Arce, A.B. Antioxidant activity of rosemary and oregano ethanol extracts in soybean oil under thermal oxidation. Ciênc. Tecnol. Aliment., v.20, n.2, p.197-203, 2000.

38. Coelho, M. G. Acute and topic anti-edematogenic fractions isolated from the seeds of Pterodon pubescens. J. Pharm. Pharmacol., v.56, n.1, p.135141, 2004.

39. Foglio, M. A.; Queiroga, C. L.; Souza, I. M. O.; Rodrigues, R. A. F. Plantas Medicinais como Fonte de Recursos Terapêuticos: Um Modelo Multidisciplinar, 2010. Disponível em:

<https://www.multiciencia.unica mp.br/artigos_07/a_04_7 .pdf.> Acesso em 25 de maio de 2015.

40. Torrenegra, R.; Bauereib, P.; Achenbach, $H$. Homoormo sanini type alkaloids from Bowdichia virgiloides.
Phytochemistry., v.28, p.22192221, 1999.

41. Marques, D.D.; Machado, M.I.L.; Carvalho, M.G.; Meleira, L.A.C.; Braz-Filho, R. Isoflavonoids and triterpenoids isolated from Pterodon polygalaeflorus. $\mathrm{J}$ Braz. Chem. Soc., v. 9.n.3, p. 295-301, 1998.

42. Fascio. M.; Mors, W. B.; Gilbert, B.; Mahajan, J. R.; Monteiro, M. B.; Santos-Filho D.; Vichenewski, W. Diterpenoid furans from Pterodon species. Phytochemistry.,v,15,p. 201-203, 1976.

43. Arriaga, A.M.C.; Castro, M.A.B.; Silveira, E.R.; Braz-Filho, R. Further diterpenoids isolated from Pterodon polygalaeflorus. J. Braz Chem Soc., v.11, p.187190, 2000

44.Braz-Filho, R.; Gotilieb, O. R.; Assumção, R. M. V. Isoflavones of Pteredon pubescens. Phitochesmistry., v.10, p.28352836, 1971.

45. Miranda, M. L. D.; Garcez, F. R.; Abot, A. R.; Garcez, W. S. Sesquiterpenos e outros constituintes das folhas de Pterodon pubescens Benth (Leguminosae). Quím. Nova., v.37, n.3, p. 473-47, 2014.

46.Sabino, K.C.; Gayer, C.R.; Vaz, L.C.; Santos, L.R.; Felzenszwalb, I.; Coelho, M.G. In vitro and in vivo toxicological study of the Pterodon pubescens seed oil. Toxicol. Lett., v.108, n.1, p.27-35, 1999a.

47.Sabino, K. C.; Castro, F.A.; Oliveira, J. C.; Dalmau, S. R.; Coelho, M.G. Successful treatment of collagen-induced arthritis in mice with a hydroalcohol extract of seeds of 
Pterodon pubescens. Phytother. Res., v.13, n.7, p.613-615, 1999b.

48. Silva, M. C.; Gayer, C. R.; Lopes, C. S.; Calixto, N. O.; Reis, P. A.; Passaes, C. P.; Paes, M. C.; Dalmau, S. R.; Sabino, K. C.; Todeschini, A. R.; Coelho, M. G. Acute and topic anti-edematogenic fractions isolated from the seeds of Pterodon pubescens.J. Pharm. Pharmacol., v.56, n.1, p.135141, 2004.

49. Carvalho, J. C. T.; Sertié, J. A. A.; Barbosa, M. V. J.; Patrício, K. C. M.; Caputo, L. R. G.; Sarti, S. J.; Ferreira, L. P.; Bastos, J. K. Anti-inflammatory activity of the crude extract from the fruits of Pterodon emarginatus Vog. J. Ethnopharmacol.,v.64, n.2, p.127- 133, 1999.

50. Coelho, L.P.; Reis, P.A.; Castro, F.L.; Gayer, C.R.M.; Lopes, C.S.; Silva, M.C.C.; Sabino, K.C.C.; Todeschini, A.R.; Coelho, M.G.P. Anti-nociceptive properties of ethanolic extract and fractions of Pterodon pubescens Benth. seeds. J. Ethnopharmacol.,v.98, n.1-2, p.109-116, 2005.

51.Euzebio, F. P. G.; Santos-Filho, J. L.; Piló-Veloso, D.; Ruiz, A. L. T. G.; Carvalho, J. E.; FerreiraAlves, D. L.; de Fatima, A. Effect of $6 \alpha, 7 \beta$-dihydroxyvouacapan$17 \beta$-oicacid and its lactone derivatives on the growth of human cancer cells. Bioorg. Chem.v.37, n.3, p.96-100, 2009.

\footnotetext{
52. Moraes, W.F;. Estudo fitoquímico e avaliação das atividades analgésica $\mathrm{e}$ antiinflamatória do extrato etanólico, frações e substância
}

isolada da casca do caule de Pterodon emarginatus VOG. (Sucupira). 2007. $102 \mathrm{f}$. Dissertação (Mestrado em farmácia).Universidade Federal de Goiás. 2007

53. Evangelista, G.L.; Coelho-deSouza, A.N.; Santos, C.F.; LealCardoso, J.H.; Lopes, E. A. B.; dos Santos, M. V.; Lahlou, S.; Magalhães, P.J.C. Essential oil of Pterodon polygalaeflorus inhibit selectro mechanical coupling on rat isolated trachea. J. Ethnopharmacol., v.109, n.3, p.515-522, 2007.

54.Leonhardt, V:" Leal-Cardoso, J. H.; Lahlou, S.; Albuquerque, A.A.; Porto, R. S.; Celedônio, N. R.; Oliveira, A. C.; Pereira, R. F.; Silva, L. P.; Garcia-Teófilo, T. M.; Silva, A. P.; Magalhães, P. J.; Duarte, G. P.; Coelho-deSouza, A. N. Antispasmodic effects of essential oil of Pterodon polygalae florus and its main constituent $\beta$-caryophyllene on rat isolated ileum. Fundam. Clin. Pharmacol.,v.24, n.6, p.749-58, 2010. 